\title{
El proyecto reformador Rivadaviano. Una propuesta didáctica para tercer año de la Escuela Secundaria
}

\section{The Rivadaviano reformer Project. A didactic proposal for the third year of High School}

\author{
Macarena Belén Jaciuk \\ macarenajaciuk@gmail.com \\ Facultad de Humanidades y Ciencias de la Educación \\ - Centro de Historia Argentina y Americana - \\ Universidad Nacional de La Plata, Argentina
}

Recepción: 10 Octubre 2020

Aprobación: 21 Noviembre 2020

Publicación: 04 Enero 2021

Cita sugerida: Jaciuk, M. B. (2021). El proyecto reformador Rivadaviano. Una propuesta didáctica para tercer año de la Escuela Secundaria. Trabajos y Comunicaciones, (53), e143. https://doi.org/10.24215/23468971e143

\begin{abstract}
Resumen: Este trabajo tiene por finalidad ofrecer ideas y material de análisis, para la realización de una planificación completa destinada a estudiantes del tercer año de la Escuela Secundaria. Teniendo en cuenta el Diseño Curricular, ${ }^{1}$ se seleccionaron fuentes del período correspondiente al gobierno provincial de Martin Rodríguez y se su principal ministro Bernardino Rivadavia (1821-1824), con el objetivo de analizar las reformas llevadas a cabo y reflexionar sobre los cambios y las continuidades con el período colonial, como también comprender las aspiraciones de los grupos reformadores y las resistencias que causaron.
\end{abstract}

Palabras clave: Reformas, Fuentes para la historia, Planificación, Tercer Año, Escuela Secundaria.

\begin{abstract}
This work tries to offer ideas and some materials, for the accomplishment of a complete planification destined to students of the third year of the High School. Taking into account the Curriculum Design, sources from the period corresponding to 1821 were selected, with the objective of analyzing the reforms carried out and reflecting on the changes and continuities with the colonial period, as well as understanding the aspirations of the reforming groups and the resistances they caused.
\end{abstract}

Keywords: Reforms, History sources, Planification, Third year, Secondary school.

\section{INTRODUCCIÓN}

Este trabajo tiene como objetivo brindar una propuesta de clase, destinada para las y los alumnos de tercer año del Colegio Secundario específicamente con el eje de Ciencias Sociales Historia del Diseño Curricular para $3^{\circ}$ año $(E S){ }^{2}$

Esta propuesta puede utilizarse tanto para un contexto áulico tradicional como virtual, es decir, es posible su realización en el marco de la educación a distancia articulando encuentros virtuales sincrónicos y asincrónicos combinando diferentes plataformas, tales como Classroom, ya sea para brindar los distintos materiales, como Meet Google o Zoom, entre otras, para dialogar con los alumnos, explicar y realizar puestas en común. Al no ser una planificación cerrada, se podrán adaptar los tiempos, según le parezca conveniente a cada docente, teniendo en cuenta las particularidades del grupo. 
Al pensar cómo debemos llevar los conocimientos al aula, cómo debemos hacer para que a las y los adolescentes puedan descubrir el valor del estudio de esta área, tenemos que tener en cuenta, que se necesita conocer a quienes tenemos en frente, conocer sus realidades, sus potencialidades, sus limitaciones, para incentivar y lograr lo mejor.

Este tipo de enseñanza no puede realizarse sin un auténtico diálogo entre el docente y los estudiantes, en el que el primero, los guie en el proceso del conocimiento con la mayor cantidad de herramientas posibles.

Que el conocimiento se encuentre estrechamente vinculado con su deseo, es un desafío para los educadores, despertar en las y los alumnos la curiosidad, a través de interrogantes que los lleven a pensar y relacionar los contenidos, con el mundo cotidiano en el que viven. Esto siempre pensado desde la perspectiva de no cometer el relativamente común error de analizar el pasado en base a anacronismos.

En esta propuesta, se abordarán las reformas, llevadas adelante por el ministro de Gobierno y de Relaciones Exteriores, Bernardino Rivadavia, durante el mandato de Martín Rodríguez, quien gobernó la provincia de Buenos Aires, entre 1820-1824.

Para ello, se tendrán en cuenta diversos instrumentos, tales como material audio visual, un padlet y fuentes escritas. Las dos primeras tienen como propósito dar a conocer el contexto general y la última, la intención de acercar, a las y los estudiantes, los documentos para tener una visión más rica como también compartir con sus compañeros las distintas interpretaciones originadas durante el proceso de análisis.

El material bibliográfico que fue tenido en cuenta para explicar el contexto histórico y para la elaboración de las diapositivas: (Cowen, 2004; Goldman, 1998; Lobato \& Suriano, 2010, pp.168-173; Ternavasio, 2015, pp.119-148).

Las fuentes utilizadas para la clase son dos: a) "Reproducción de los fundamentos expresados por el Ministro de Gobierno Bernardino Rivadavia, en la Sala de Representantes, para suprimir los cabildos de la provincia" (Goldman, 1998, p. 173 ) y b) "La reforma eclesiástica" (Cowen, 2004, p.86)

Está clase podría desarrollarse a continuación de la clase correspondiente a los temas de: "La inestabilidad en la organización de los nuevos Gobiernos. Guerras Civiles”. (Bracchi, 2008, p.168), según lo establecido por el Diseño Curricular.

\section{Propósitos}

Esta propuesta busca generar un espacio de análisis y diálogo, a través de la interpretación de fuentes y la utilización conjunta de otros materiales. Al mismo tiempo, problematizar las reformas y las posturas tomadas por los sujetos en el período estudiado.

\section{OBJETIVO GENERAL}

Que las y los estudiantes conozcan las distintas reformas emprendidas durante el período denominado la "Feliz Experiencia" y que a través de los materiales brindados puedan realizarse preguntas, reconstruir el contexto y relacionarlo con el tiempo presente.

\section{OBJETIVOS ESPECÍficos}

- Conocer el contexto en el que se desarrolla el proyecto Rivadaviano.

- Distinguir los distintos sujetos que aparecen vinculados al proceso.

- Comprender las diferentes reformas como así también sus causas y sus consecuencias.

- Identificar las resistencias ante las distintas normativas. 


\section{Desarrollo: Recursos}

Para esta propuesta se utilizará un fragmento del video "Capítulo III. Unitarios", que forma parte de la serie "XIX. Proyectos de Nación" de Canal Encuentro, que tendrá como objetivo dar a conocer un panorama general del contexto histórico y de las distintas reformas. ${ }^{3}$ (ver anexo $\mathrm{n}^{\circ} 1$ )

Además, se trabajará con una pizarra virtual o padlet, ${ }^{4}$ (ver anexo $\mathrm{n}^{\circ} 2$ ) y dos escritas citadas (ver anexo $n^{\circ} 3$ y 4$)$

\section{Desarrollo de la Clase}

La clase se iniciará con el video, que puede ser visto durante la misma o enviado el link con antelación. Una vez finalizado, se procederá con la elaboración de preguntas a modo introductorio del tema. Se espera que los estudiantes puedan dar su apreciación y explicar la información proporcionada por el mismo.

Luego, se presentará el padlet, que deberán completar entre todos, con el fin de reconstruir el período desarrollado. Al terminar esta actividad se podrá comentar, e incorporar alguna explicación en relación a la misma.

A continuación, se les indicará la actividad con el documento escrito. Para eso, se les pedirá que cada uno seleccione una fuente.

Para finalizar se llevará a cabo una puesta en común, para compartir la actividad realizada. De esta manera, todos podrán acceder mediante los comentarios de sus pares, a la fuente que no trabajaron.

Este período también suele ser conocido como la feliz experiencia, denominación concedida por los contemporáneos, haciendo referencia a la armonía y al orden imperante en Buenos Aires en oposición a la época turbulenta vivida años anteriores. No obstante, tuvo una fugaz duración, su impronta y continuidad en algunos casos no deja de sorprender.

El experimento político desplegado en Buenos Aires entre 1821 y 1824, conocido, según la expresión de la época, como la feliz experiencia, no llegó a concretarse en una constitución escrita. A pesar de que la Sala de Representantes se declaró extraordinaria y constituyente el 3 de 1821, y se otorgó un año de plazo para dictar una constitución, no fue sancionada carta orgánica alguna en el ámbito provincial hasta 1854. (Ternavasio, 2015, p.141)

La tardanza en la sanción de la Constitución provincial, estuvo asociado, como sostiene la autora, fundamentalmente con el papel que tomó la provincia de Buenos Aires, en relación al resto:

La centralidad que asumía Buenos Aires en el escenario global del ex virreinato la diferenciaba del resto de las provincias, para quienes dictar su propio reglamento constitucional significaba consolidar sus instituciones frente a cualquier intento de nacionalización del cuerpo político. Buenos Aires, en cambio, se sentía heredera del poder central caído a la vez que protagonista de cualquier emprendimiento constitucional a nivel nacional. (Ternavasio, 2015, p.141)

Las reformas tuvieron como objetivo crear y consolidar bajo un signo modernizador las estructuras política y administrativas de la provincia, en un lapso muy corto de tiempo. Se pretendía instaurar un clima de orden y armonía. Las mimas abarcaron distintos aspectos, tales como la política, la economía, la sociedad y la cultura.

En el plano político, podemos mencionar, en primer lugar, la sanción de la ley electoral de 1821, que estableció nuevas normativas para la elección de diputados para la Sala de Representantes y estos a su vez, al gobernador. Se aumentó el número de electores, ya que, sin requisito alguno, salvo el de ser libre, podían votar todos los varones.

Otra ley sancionada en ese mismo año, fue la supresión de los cabildos de Buenos Aires y Luján, que pasaron a ser instituciones municipales. Esto opacó notablemente su poder, en beneficio de las autoridades provinciales. 
Se reconfiguró la Justicia, estableciéndose una división entre una justicia de primera instancia y otra de paz, la primera se caracterizó por ser letrada y paga, mientras que la segunda, gratuita. El poder legislativo recaía en la Sala de Representantes.

La ampliación de la frontera, ocasionó conflictos con los indígenas. Si bien el gobernador concertó la paz, no cumplió sus promesas, esto generó el descontento de las comunidades, manifestado a través de sucesivos malones. No es algo menor resaltar que el sector dominante política y económicamente en la provincia desde la caída de las autoridades nacionales fueron los hacendados. Martin Rodríguez, no solo fue un destacado militar sino también un eminente representante de este grupo socioeconómico que tuvo una posición hegemónica en la provincia.

En 1822 se creó la Bolsa Mercantil, el Banco de Descuentos, y en 1824, se solicitó un empréstito a la Baring Brothers Co, con el objetivo de destinarlo a la construcción de infraestructura, como puertos, obras sanitarias. Como garantía de dicha deuda, se establecieron las tierras públicas.

También, en ese año se aprobó la Ley de enfiteusis, que pretendía obtener mayor ganancia de las tierras a mediante la instalación de colonos.

En el plano social, "el gobierno procuró que muy pocos ámbitos sociales escaparan al ímpetu reformista y ordenador." (Cowen, 2004, p.62)

Con la ley de reforma militar, se buscó la reducción del gasto público, a través de la disminución del ejército. El argumento no solo fue económico, sino que se justificaba argumentando que la guerra de independencia ya no se libraba en territorio argentino al propio tiempo que no se dejaba de recordar que las autoridades nacionales habían cesado.

Con respecto a la Iglesia, el objetivo era centralizar y controlar esta institución. La existencia de las distintas órdenes, hacía difícil concretar dicho proyecto. Las medidas adoptadas en perjuicio de estas fueron: la eliminación del diezmo, la incorporación de algunos de sus bienes al Estado, se produjo la secularización de los regulares y el Estado se comprometió a solventar los gastos de la Iglesia, entre otras.

La moral y la visión rigurosa de quienes emprendieron este proyecto, nada tenía que ver con lo religioso, sino planteadas desde el laicismo, relacionadas con las corrientes liberales y la ilustración.

La obra pública, uno de los ejes de la política rivadaviana, debía ser ejecutada por profesionales que posean formalmente el título de ingeniero. Se redujo al mínimo el número de días feriados y persiguió los juegos que causaban más estragos en la sociedad, como los nocivos y vergonzantes juegos de carnaval. Se condenó enérgicamente la mendicidad y la embriaguez. (Cowen, 2004, p.63)

En 1823, se fundó la Sociedad de Beneficencia:

Quizás de todas las sociedades y academias creadas por el influjo del ministro Rivadavia, la Sociedad de Beneficencia sea la más importante, ... por un lado por la incorporación de mujeres de la elite a responsabilidades que hasta ese momento no asumían públicamente y por otro, su llamativa perdurabilidad ... Se confiaba a la institución la dirección y la inspección de la Escuela de Niñas, Casa de partos Públicos y Ocultos, cuya existencia no llegó a concretarse - el Hospital de Mujeres, el Colegio de Huérfanos y de todos los establecimientos de esa índole. (Cowen, 2004, p.67)

Son numerosas las medidas que giraron en torno a lo cultural, ya que se dio un gran impulso a las artes y las ciencias, se podrían destacar, la creación de la Universidad de Buenos Aires en 1821, el Archivo General también en ese año. En 1823, la inauguración del Museo de Historia Natural. Como la creación de sociedades literarias. Entre las publicaciones periódicas más influyentes y que acordaban en general con el gobierno, se encontraban, El Argos de Buenos Aires y La Abeja Argentina.

$\mathrm{Al}$ reflexionar sobre los resultados de tales reformas, podemos decir, que, si bien desde el gobierno se inten tó conducir a la sociedad hacia un estado de progreso y modernidad, como aquella elite porteña entendía conveniente, adoptando a Europa como modelo, no tuvo la respuesta esperada y no llegaron las estructuras a transformarse acabadamente. Quedó claro que, para avanzar en ese proceso, era necesario desprenderse de los 
antiguos resabios coloniales y dar paso a nuevas conductas. Dejar de lado a sectores, como las comunidades indígenas, para recibir a los nuevos colonos. Como también, el corrimiento de lo religioso, en pos de lo secular.

Aunque el impacto del proyecto rivadaviano fue limitado y la desproporción entre los objetivos de máxima propuestos por la elite y los cambios realmente producidos fue amplia, la transformación que en menos de un quinquenio afectó al Estado bonaerense no dejó de ser notable. (Goldman, 1998, p. 177)

\section{ACTIVIDADES}

La primera actividad consiste en ver el material audio visual y dar cuenta de lo observado. Para guiar en ese proceso, se realizarán las siguientes preguntas:

¿Quiénes decidieron emprender las reformas?

¿Por qué sintieron esos sujetos la necesidad de emprender ese proyecto?

¿Qué ideas inspiraron la implementación de las medidas?

¿Cuáles fueron los obstáculos que aparecieron durante todo el proceso?

La segunda, es una actividad grupal, en la cual se pretende que las y los estudiantes completen conjuntamente, la información requerida en el padlet. ${ }^{5}$

La tercera, es el trabajo con la fuente. Cada uno seleccionará un documento. Se espera que la actividad les permita a los estudiantes ir de lo particular a lo general. Es decir, en un primer momento, realizaran una lectura guiada y minuciosa del documento, en la que puedan comprender de que se trata, para luego realizar una lectura más crítica y en la que puedan expresar su postura y la comprensión del tema.

Esta acción de interpretar, criticar y desnaturalizar la aplicamos no solo sobre acciones de otros sujetos, sino también sobre nuestras propias acciones y pensamientos, dado que cada uno de nosotros somos parte de la Historia, lo que implica que las decisiones que tomamos, marcar cierto rumbo y también podemos observarlas y criticarlas. La Historia en este caso tendría, además, la función de autoconocimiento.

Si conozco el pasado y los grandes modelos del pasado, si tengo cultura histórica, si averiguo quiénes fueron los que me precedieron y con quiénes se midieron realmente o con quienes fantasearon, sabré mejor qué clase de individuo soy o aspiro a ser en la sociedad que me toca vivir, qué clase de individuo no quiero ser si otros antes que yo lo fueron. (Serna, 2006, p.74)

Finalmente, la puesta en común, permite que los estudiantes den a conocer sus ideas, se fomenten pequeños debates, valorando las miradas de los otros. Como también entender que, aun trabajando el mismo documento, el resultado puede ser diferente.

Las consignas a trabajar con las fuentes son:

Fuente 1: Fundamentos para suprimir los Cabildos

1. Marcar en el documento con distintos colores los siguientes datos: Sujetos que aparecen en la fuente:

a) Quién expone los argumentos a favor de la eliminación de los Cabildos.

b) A quienes se les explica.

c) Individuos mencionados en los fundamentos.

d) Tipos de gobiernos citados.

e) Ejercicio de la Soberanía.

f) Fecha de producción del documento

2. ¿Por qué deberían suprimirse los Cabildos según lo expresado en el documento?

3. Elegir algunas de las medidas y explicar qué similitudes o diferencias pueden encontrar en relación a la actualidad. (Puede ser otra a la que se trabajó en la fuente)

Fuente 2: Ley eclesiástica 
1. Marcar en el documento con distintos colores los siguientes datos:

a) Quiénes elaboran las medidas.

b) Afectados por la nueva reglamentación.

c) Edificios que se mencionan.

d) Fechas que aparecen.

e) El medio utilizado para dar a conocer las medidas.

2. Explicar en qué consistió la nueva reglamentación y de qué forma se vieron afectados los miembros de las comunidades religiosas.

3. Elegir algunas de las medidas y explicar qué similitudes o diferencias pueden encontrar en relación a la actualidad. (Puede ser otra a la que se trabajó en la fuente)

\section{REFERENCIAS}

Bracchi, Claudia. (2008). Diseño Curricular para la Educación Secundaria $3^{\circ}$ Año. Dirección General de Cultura y Educación de la Provincia de Buenos Aires. Recuperado de http://servicios.abc.gov.ar/lainstitucion/organismo s/consejogeneral/disenioscurriculares/documentosdescarga/dc_ter1_08_web.pdf

Canal Encuentro. (2010). XIX Los proyectos de Nación. Recuperado de: http://encuentro.gob.ar/programas/serie/80 91/1339?temporada=1

Cowen, Pablo. (2004). Martín Rodriguez. Los avatares de una élite reformista. La Plata: Archivo Histórico de la Provincia de Buenos Aires "Dr. Ricardo Levene".

Goldman, Noemí. (1998). Nueva Historia Argentina. Revolución, República, Confederación 1806-852. Recuperado de https://ens9004-infd.mendoza.edu.ar/sitio/nueva-historia-argentina/upload/Nueva_Historia_Argentina_ Tomo_3.pdf

Lobato \& Suriano (2010). Nueva Historia Argentina. Atlas Histórico. Buenos Aires: Sudamericana.

Serna, Justo. (2006). Sobre la utilidad y el perjuicio de la historia para la vida adolescente. Clio \& Asociados, 9-10,71-83.

Ternavasio, Marcela. (2015). Historia de la Argentina 1806 -1852. Buenos Aires: Siglo Veintiuno Editores.

(https://es.padlet.com/)

(https://www.youtube.com/watch?v=O_pU14mlFk)

(https://es.padlet.com/macarenajaciuk/ActReformasRivadavianas)

\section{Anexo 1}

Sinopsis Serie "XIX. Los proyectos de Nación": Durante el siglo XIX, distintos grupos de la sociedad argentina impulsaron proyectos para la reconstrucción de una nación nueva. ¿Cuáles eran los ideales de quienes propulsaron la Revolución de Mayo? ¿Por qué hubo gente dispuesta a dar la vida por esas ideas? Un recorrido desde la revolución que inició la emancipación hasta la formación de ese país pensado, planeado y soñado.

Sinopsis Capítulo "Capítulo III. Unitarios": El proyecto del unitarismo tuvo como papel central en la cultura política argentina del siglo XIX. Durante la década de los veinte, sus adeptos proponían organizar el país de forma centralizada desde Buenos Aires. Su fracaso y su legado. 


\section{Anexo 2}

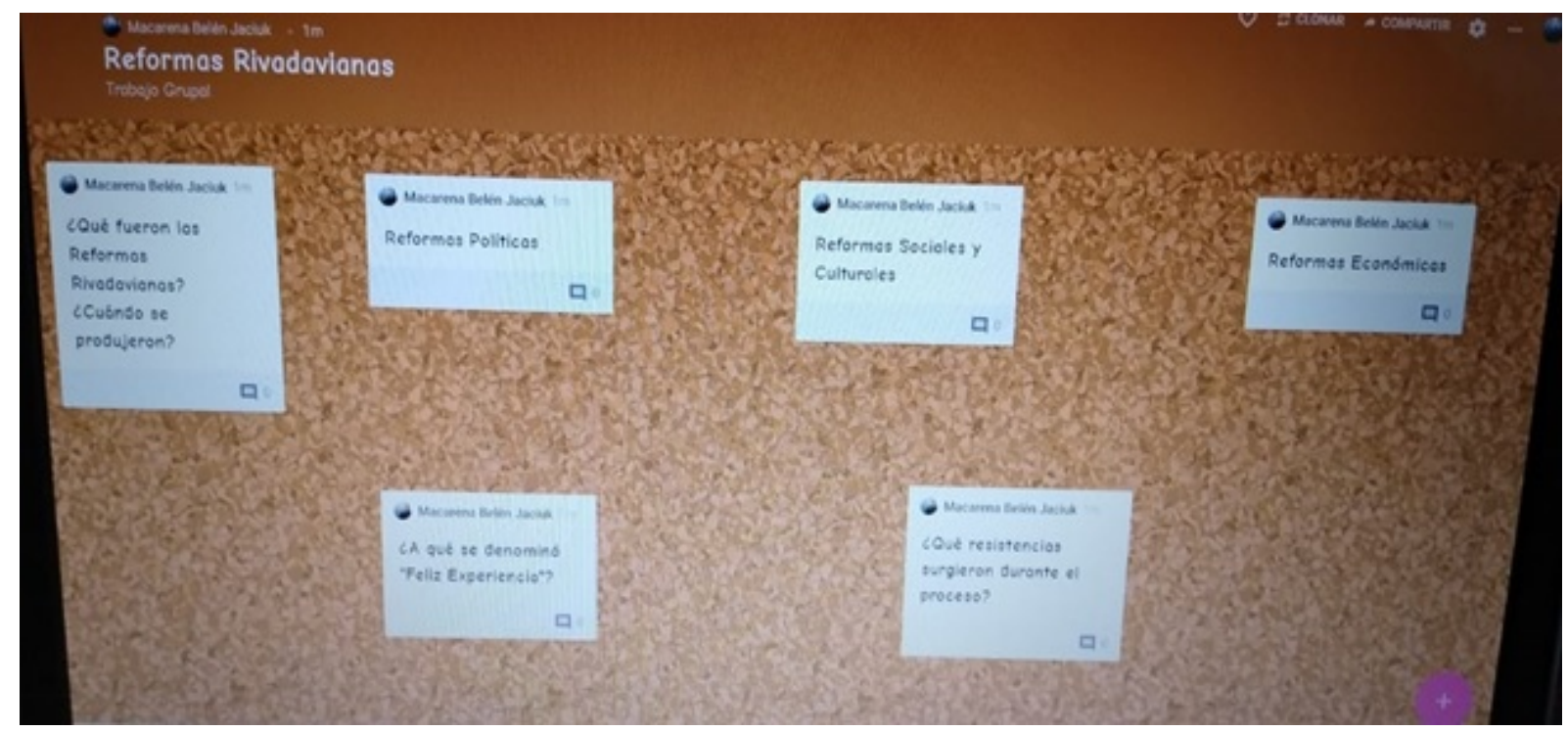

\section{Anexo 3}

Reproducción de los fundamentos expresados por el Ministro de Gobierno Bernardino Rivadavia, en la Sala de Representantes, para suprimir los cabildos de la provincia

"... cuanto en España era el reynado de Carlos III y Felipe V, y habiendo marcado el carácter, facultades y modificaciones de los Cabildos do América establecidos por el Gobiemo Peninsular dijo que tan nocesarios eran los Cabildos en aquel orden, como innecesarios al presente; que en un Gobiorno Monárquico absoluto era el que la soberanía nacional estaba personificada al individuo que la ejercía por títuio de sucesión, era indispensable reservarse un resto de autoridad para los Pueblos depositándola en manos de los que en aquel orden obtenian su representación, poro que este establecimionto era incompatibie con un Gobierno Representativo en que esa autoridad suprema ha retrovertido a la sociedad, y exerce con toda la plenitud de un sistoma liberal por medio de aquellas autoridades que tienen la viva representación los Puebios confusiones reaies que les ha circunscrito la naturaleza del Gobierno actual y los pactos sociales; que en ese estado aparecen los Cabildos sin una atribución real, y útil al público...".

Extraido de: Acuerdos de la $\mathrm{H}$. Junta de Representantes de la Provincia de Buenos Aires (1520-1821). Publicaciones del Archivo histórico de la Provincia de Buenos Aires, La Plata, 1932, vol. I, sesión del 5 de diciembre de 1821. 


\section{Anexo 4}

MAR'TIN RODRIGUFT

\section{L2 reforma oclesiástica}

La llamada reforma religiosa fue quizds Lo que despertó las mayores criticas al gobierno, tanto que incluso el propio Gregorio Tagle, justificó sus levantamientos alegando una politica anticristiana por parte del gobierno. Aquí presentamos algunas de las medidas que el gobicrno de Rodrfguez anumció por el Registro Oficial.

"1. Los religiosos que habitan en el edificio llamado de la Recoleta, pasarán a su elección o al convento llamacio de La Obsetvancia en esta ciudad, o al de La Recolección de San Pedro.

2. Los preindicados religiosos llevarán consigo todos los útiles y muebles de sus respecrives usos.

3. El edificio llamaklo de La Recoleta, $y$ los mucbles cxistentes en el, a excepción de los expresados en el artículo anrerior, quedan bajo las inmediatas órdenes del Ministro Secretario de Cubierno

4. Queda destinado a cernenterio públiço el edificio prenominado

5. El Ministro Secrecario de Gobierno queda facultado para expedir todas las órdcnes, que han cfecrivo el más pronto cumplimiento de cste decreto, con arreglo al 13 de diciembec de $1821 \ldots$.

\section{Rodríguez-Bernardino Rivadavia}

La reforma rcligiosa: Registro Oficial. Libro dos. No. 19. Tomó uno. Buenos Aires. Julio cuatro de 2822, Págs. 231 a 233.

\section{Notas}

1 El contenido abordado es tomado del diseño curricular jurisdiccional de la provincia Buenos Aires, pero el mismo se articula con los lineamientos establecidos por los núcleos prioritarios de aprendizaje federales. Si bien la propuesta está dirigida a docentes de Historia de 3er año en la Prov. de Bs As, podría ser utilizado en otras provincias ajustado a sus diseños curriculares.

2 Como se mencionó anteriormente si bien se utiliza el diseño curricular curricular jurisdiccional de la provincia Buenos Aires, podría ser utilizado en otras provincias ajustado a sus diseños curriculares.

3 El fragmento seleccionado corresponde al bloque de Reformas Rivadavianas. Se puede ver directamente el recorte ya realizado en: https://www.youtube.com/watch?v=O_pU14mlFk

4 Padlet, es una herramienta para elaborar murales digitales online, que posibilita el trabajo en conjunto. Para crear una pizarra virtual se sugiere ver: ¿Cómo hago para crear un mural digital con Padlet? https://www.youtube.com/watch? $\mathrm{v}=\mathrm{zLZRy} 1 \mathrm{sNiz} 4$

5 Padlet elaborado a modo de ejemplo: https://es.padlet.com/macarenajaciuk/ActReformasRivadavianas 